\title{
Mathematics as a Common Language to Our Civilization
}

\author{
Carson Lam Kai Shun"
}

University of Hong Kong, Pok Fu Lam, Hong Kong

DOI: $10.36347 /$ sipms.2020.v07i10.006

| Received: 29.09.2020 | Accepted: 13.10.2020 | Published: 15.10.2020

*Corresponding author: Carson Lam Kai Shun

Abstract

Original Research Article

\begin{abstract}
Mathematics surrounds us and has existed since ancient times. Numerals originated from performing daily tasks that required conceptual counting. Later, symbols began to appear and had to be memorised. Over time, these formulas were simplified and transformed into notations. The collection of these symbols and notations became words, which gradually developed into a language. Indeed, mathematics can be used as a rational tool to link all kinds of knowledge. Throughout history, mathematics has been connected with all past human endeavours, and these achievements were composed to form different cultures. In addition, modern civilisation only has one common type of mathematics-in terms of symbols - to depict the world's natural phenomena. Therefore, mathematics is a common language for different nations and cultures. Taking a step further, this arguably implies that we have one creator, Christianity GodJehovah.

Keywords: mathematics, endeavours, symbols, language.

Copyright $\left({ }_{0} 2020\right.$ The Author(s): This is an open-access article distributed under the terms of the Creative Commons Attribution 4.0 International License (CC BY-NC 4.0) which permits unrestricted use, distribution, and reproduction in any medium for non-commercial use provided the original author and source are credited.
\end{abstract}

\section{ORIGIN OF SYMBOLS}

The subject 'mathematics' dates back to ancient times when people starts tocount. Mathematics has many symbols and notations, which are commonly used today. The question is, where did these symbols originate? According to Mazur [1], the word 'mathematics' originated from the Greek word "token" or "token of identity". It combines the root words: sum (together) and the ballo (to throw) or precisely "to put together". The ancient meaning is defined as, "a proof of one's identity or one's relationship to another". When something is divided into two and is given to one another, these two pieces fit together perfectly and identify their relationship. At the same time, notations can come from shorthand or abbreviated terms. For example, the notation "+" is the shorthand Latin word for et. That said, others believe that symbols originated from objects surrounding people. Interestingly, ancient people used visual arts to memorise the types of animals that they were able to hunt. Gradually, these arts developed numerical ideas, which were then simplified and transformed into symbols or notations. Thus, this author proposes that mathematical symbols are related to conceptual numeral objects that can be pre- sented as visual arts. They are then shortened to give suitable abbreviations whenever necessary. The following section will demonstrate how these ancient symbols or nota- tions contributed to the development of modern mathematics.

\section{LITERATURE REVIEW}

One of the most famous ancient visual works of art is known as the Chauvet Cave paint- ings. It was discovered by three French speleologists in December, 1994 [2]. The paintings mainly consist of 13 species of animal, which were used by hunters to avoid danger and to find food. According to Merzbach and Boyer in 2011 [3], the number, magnitude, and form of the paintings related to difference species, inequality in size, and unlikeness in appearance. Gradually, they found that there was a similarity in the counting of a wolf, sheep, and tree - the (conceptual) uniqueness [2]. Finally, they attempted to use a symbol "|" - the numeral "one" - to represent this relationship, the only one real object, and the idea "one". When the symbol is simplified and abbreviated, it becomes the modern numeral notation " 1 ". Indeed, at the stage of rep- resenting numerals by symbol, there would have been many alternatives and options. This study will review the four most famous civilisations in the world: Egypt, Babylonia, India, and mainland China. 


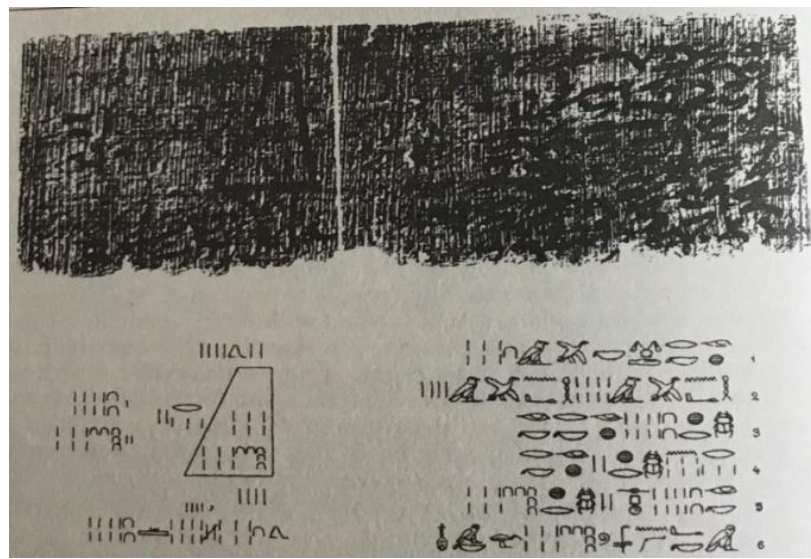

Counting Symbols among Historical Civilisation Egypt

Reproduction of a portion of the Moscow Papyrus, showing the problem of the volume of a frustum of a square pyramid, together with hieroglyphic transcription [3].

Egypt is well known for its contributions to geometry - the elementary congruence relation in the measuring device together with the beginning of a congruence theory and the idea of proof in geometry [3]. The problem was that Egypt had no clear cut relationship between exact amounts and approximations in geometry - just like Babylonia. This issue will be discussed in a later section. These numerals were developed to solve problems such as 'Heap', 'Geometric' and 'Slope' questions etc. The following image shows a numeral system that was used by the ancient Egyptians:

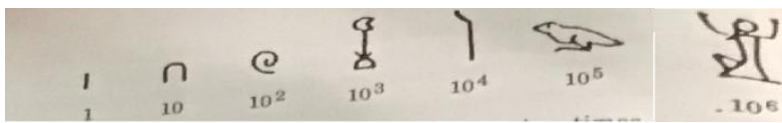

Egyptians’ numeral system (Gullberg, 1997) [4]

When discussing Egyptian numeration [4], one usually refers to hieroglyphics of some 5,500 years ago. Hieroglyphics were used as a numerical grouping system where basic numbers can were added together to express complicated numbers.

Firstly, in the hieroglyphics system, a vertical line means the number one; a heel-bone is 10; a coiled rope is 100; a lotus blossom is 1,000 ; a bent finger is 10,000 ; a tadpole is 100,000; and a kneeling genie with raised arms is 1,000,000. There are also some rules for the added numbers. They are the following:

1. Each symbol is stepped one at a time and starts from one to nine times;

2. The order of the symbols is not important, but they are likely to be of descending order either from left to right or from right to left;

3. It is a ten-based number system known as an additive decimal system.

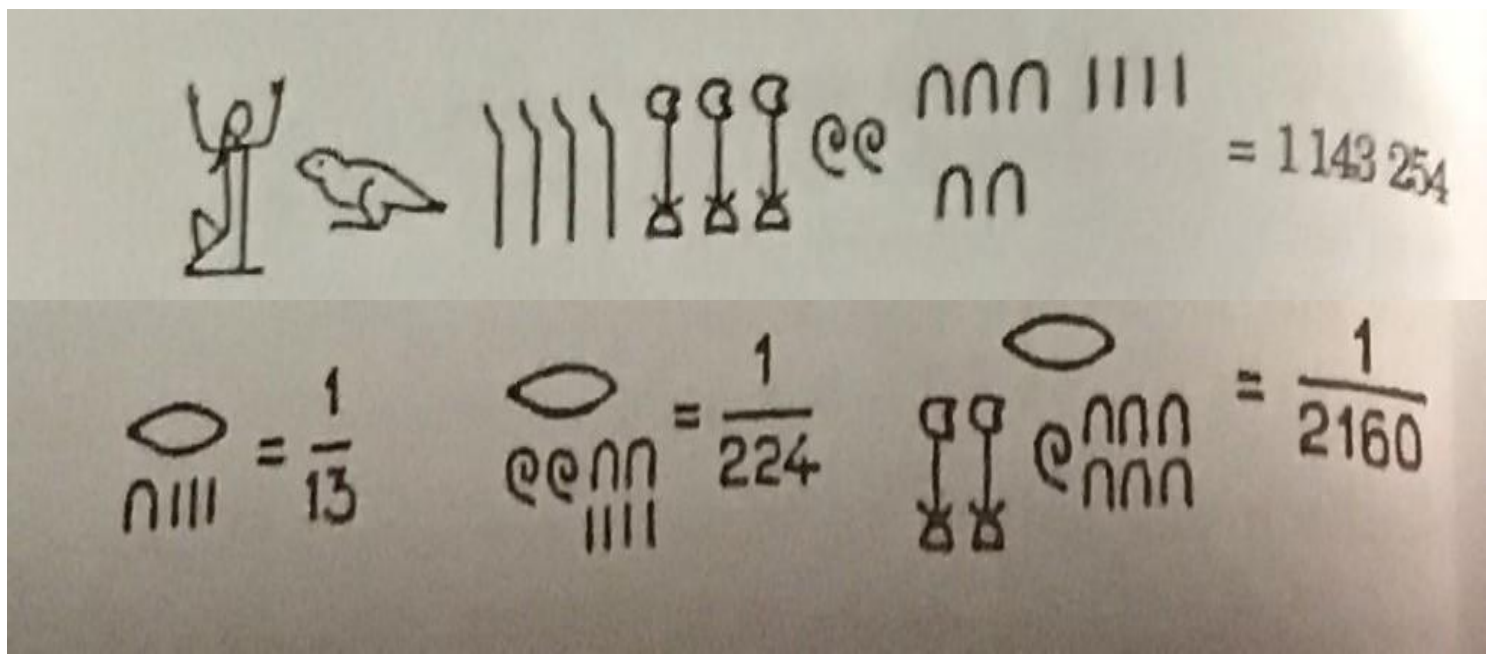

Ancient Egypt's representation of large number and fraction [4]

The ancient Egyptians always used so-called unit fractions with number 1 as the numera- tor together with the symbol - an open mouth as the denominator [4].

\section{Babylonia}

The Babylonians are best known for their contribution to numeral approximation. They also developed sexagesimal fractions to find the square root of two from the approximation view [3]. In fact, they are superior to the ancient Egyptians in the sense that principle of position can be extended to cover both fractions and whole numbers. To be precise, they had the notation which has the same meaning for $2(60)+2$, $2+2(60)^{\wedge}-1,2(60)^{\wedge}-1+2(60)^{\wedge}-2$ two successive positions. That said, they also faced the same problem of distinguishing exact and approximation values [3]. 

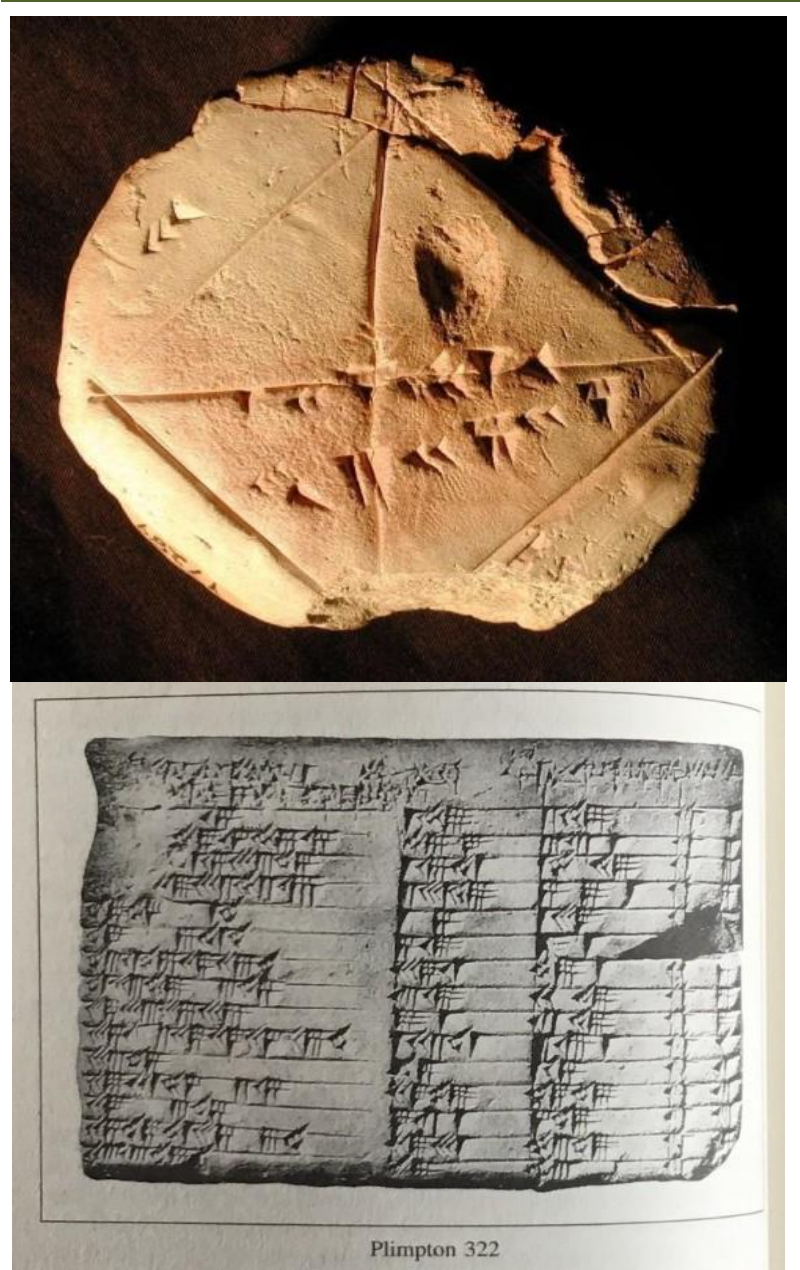

Plimpton 322 and YBC 7289 Clay tablet (Merzbach \& Boyer, 2011) [3]

Plimpton 322 is a Babylonian clay tablet that shows how they developed an approximation method for finding the answers (approaching) to irrational numbers in calculating the area of a paddock. However, they may not have established the concept of number by using the following: when a right-angled isosceles triangle with two sides is equal to one unit, the length of the hypotenuse is just the square root of 2 . When continuing the process of adding a unit length as another side, one may even find the square root of numbers from 1 to 17 [5]. This is because the idea of irrational number was not developed until around 300400 B.C. by Theaetetus and Eudoxus. It may be suggested that the Babylonians were only one step away from discovering the concept of irrational number.

Another difference is that -- unlike the Egyptians - the Babylonians were familiar with the Theorem of Thales, which was formulated more than a millennium earlier than Egypt. This is the only contribution of the Babylonians in pure geometry, as most of geo- metric theorems come from the applied arithmetic in the topic. In belief, the Babylonians had two systems of numeration: a sexagesimal system for astronomy together with a decimal system for daily usage.

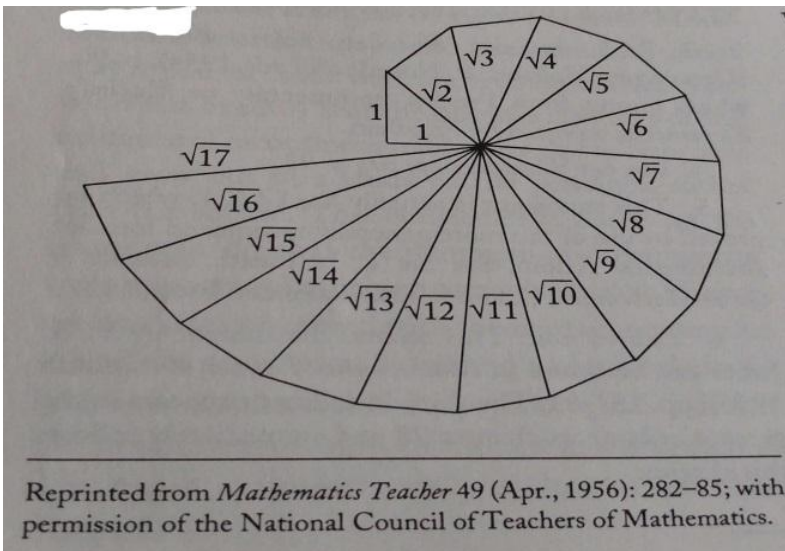

Theodorus of Cyrene (400 B.C.) etc showed the irrationality of non-square integers from 3 to 17 . The origin may come from Euclid, Book X, Proposition 8 [5]
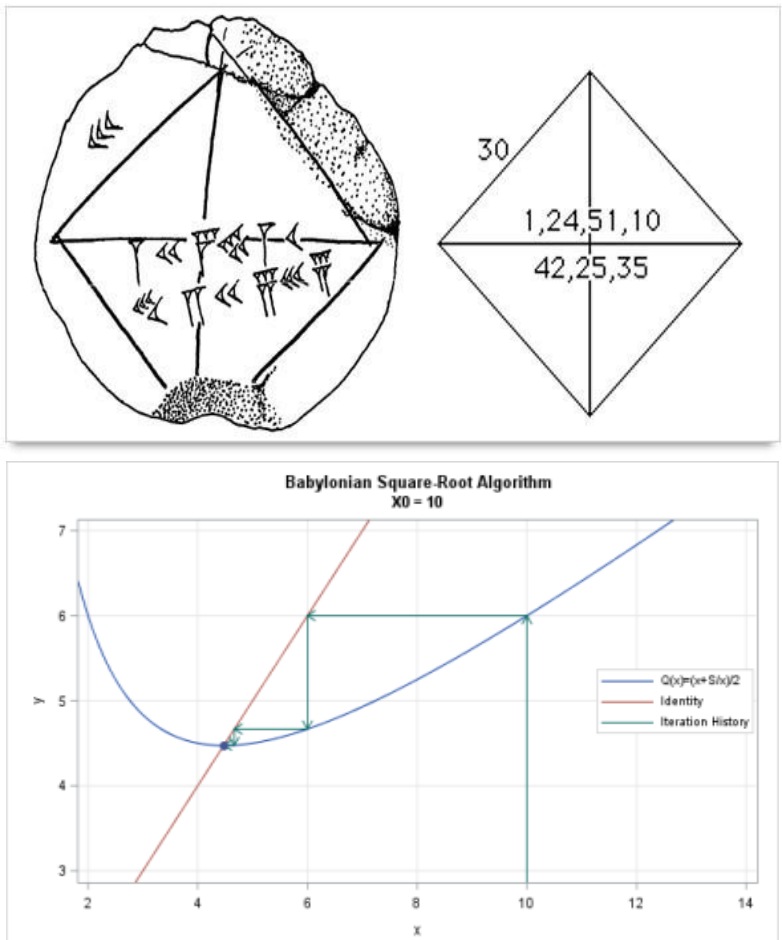

Triples of values in YBC 7289 and its numeral approximation convergence

There are further explanations about YBC 7289 and Plimpton 322. YBC 7289 explains that as early as 1800 - 1600 B.C., the Babylonians knew the ratio of unit length sides to the diagonal of a square where the length is equal to the square root of 2 . In fact, they used Archytal's Method (428 - 365 B.C.) for the approximation [3]; Let " $a$ " be the expected answer. Suppose there was an " $\mathrm{a}_{1}$ " which is the first guess, then one may have the following steps:

Step 1: $b_{1}=a / a_{1}$ (makes either one extremely big while the other is extremely small);

Step 2: $a_{2}=1 / 2\left(a_{1}+b_{1}\right)$ (calculates the arithmetic mean of them);

Step 3: $\mathrm{b}_{2}=\mathrm{a} / \mathrm{a}_{2}$ (starts the process);

Step 4: Continuing the process and one may get $a_{n}=\left(a_{n-}\right.$ $\left.{ }_{1}+b_{n-1}\right) / 2$ and $b_{n}=a / a_{n}$ until the desired answer. 
However, the Greek philosopher Pythagoras, who had lived around 600 B.C. established the famous Pythagoras Theorem. Tablet YBC 7289 tells us that ancient Babylonians had a good approximation to square root of 2 to 1$) ; 23,51,10$. With reference to the above finding, this author suggests that the Babylonians could list out a pretty ordered three sides of a rightangled triangle. They may also knew a lot about the relationships between these sides. However, they had difficulties in linking those applied arithmetic results to formulate the conclusively pure geometric Pythagorean's Law. In other words, they were weak in transforming applied values into pure mathematical rules.

Ref:

https://johncarlosbaez.wordpress.com/2011/12/02/babyl on-and-the-square-root-of-2/

https://www.maa.org/press/periodicals/convergence/the -best-known-old-babylonian- tablet https://ipch.yale.edu/news/3d-print-ancient-history-onemost-famous-mathematical-texts- mesopotamia

India

A major merit of India in numeral development is that the nation acted as a knowledge hub for exchanges between Eastern and Western culture. One case study is the number "zero" -- the introduction of a notation for a missing position after one to nine symbols [3]. Most believe that zero originated from Greece during the Alexandria period. It was then transmitted to India after the establishment of the decimal position system. When Indians started using the symbol "zero" - a round goes egg notation, one will achieve the modern system of numeration for integers.

The Hindu system is combined of three other ancient principles:

1. A decimal;

2. A positional notation;

3. Each of the ten numerals is ciphered;

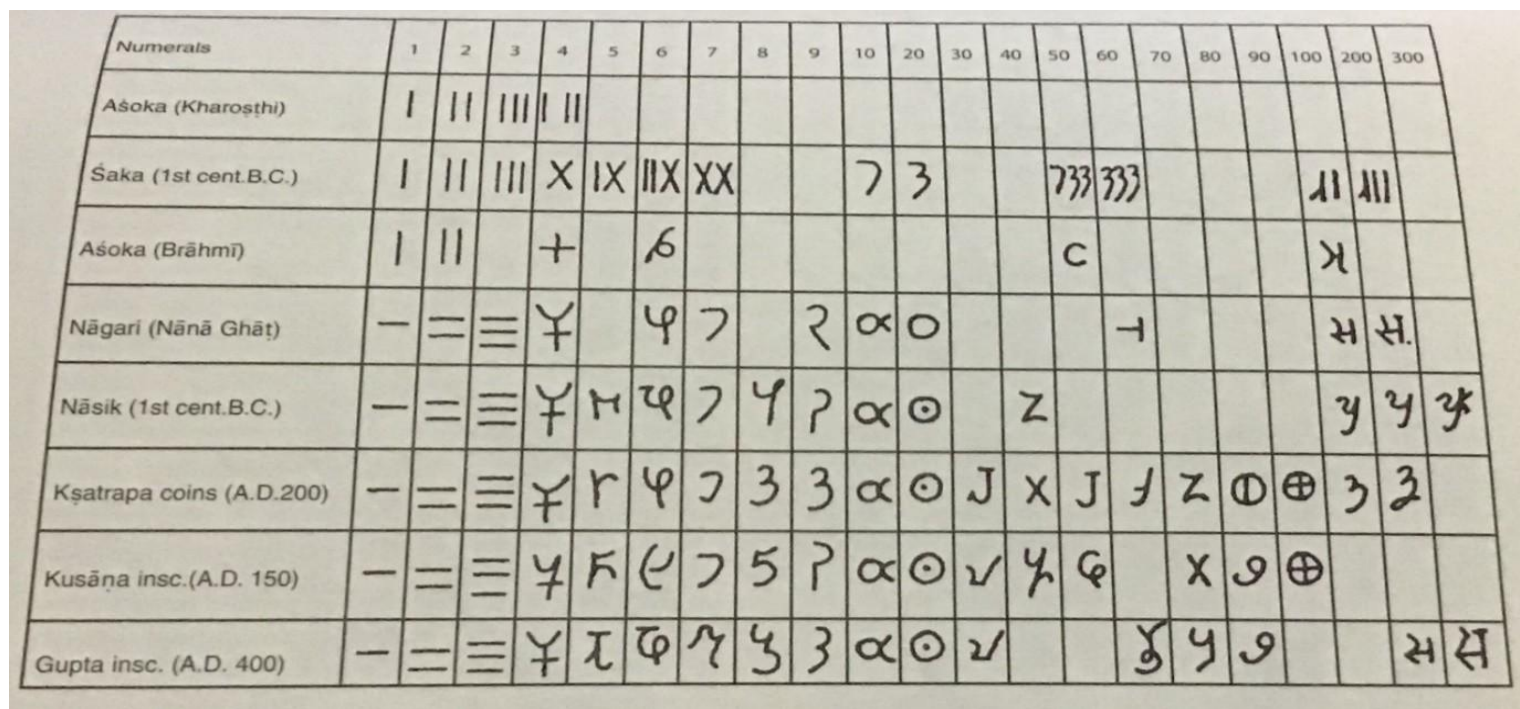

Early Indian non-place-value numeral forms [6]

However, none of the above principles originated from the Hindus. That said, once they had passed through India, the modern numeration system was founded. Indeed, there were various Indian tradition, these include computational techniques, astronomical and calendric concepts, decimal placevalue numbers. There were also geometrical relations with Greek number systems and Roman Empire (plane trigonometry of chords, geocentric cosmological models, planetary eccentrics and epicycles, and astrological motivations etc) [6]. Clearly, this is the most fitting case study for the successful exchange between Eastern and Western civilisation.

Certainly, the origin of the number "zero" has been the subject of much debate:

1. The Greek letter omicron - the initial letter in the word "ouden" or "empty";
2. The early zero symbols in Greek sexagesimal fractions were round rather than oval in shape;

3. In the 15th century, the Byzantine Empire used the zero sign, which is quite unlike an omicron. It was either an inverted form of the small letter " $h$ " or appeared as only a dot [3].

The symbol zero most likely originated from Greece (since this is the most possible evolution based on the available evidence) together with the three other ancient principles. The purpose of developing such a system was to determine a person's position on Earth when being observed from the universe - a mathematical physics problem [6]. 


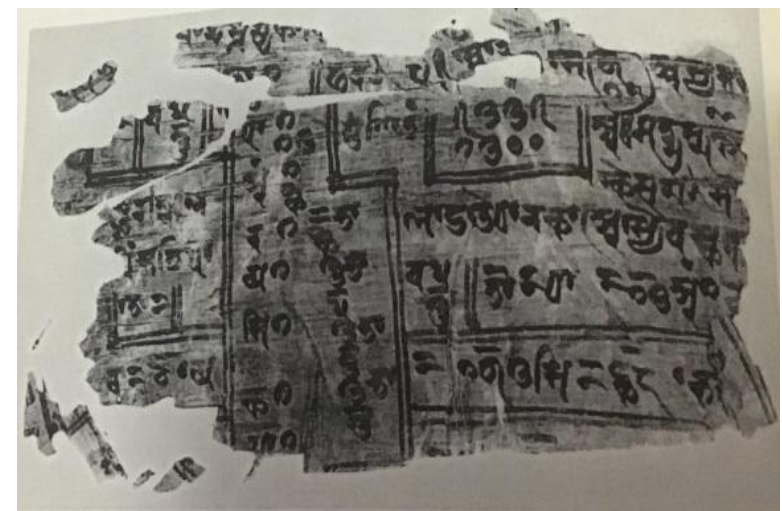

Place-value numerals in the Bakhshali Manuscript (Katz, 2007) [6]

\section{Mainland}

There were numerous mathematical achievements in ancient Mainland that are highly related to numeral developments. It is well known that one of ancient Main- land's numeral symbols was in the form of a knot. This knot is very similar to those that were used in Peru. Indeed, the so-called knot numerals were finally transformed into an ancient Mainlander magic square. It finally turned into the problem of solving ancient Mainland's simultaneous system of linear equations. Mainland Magic Square.

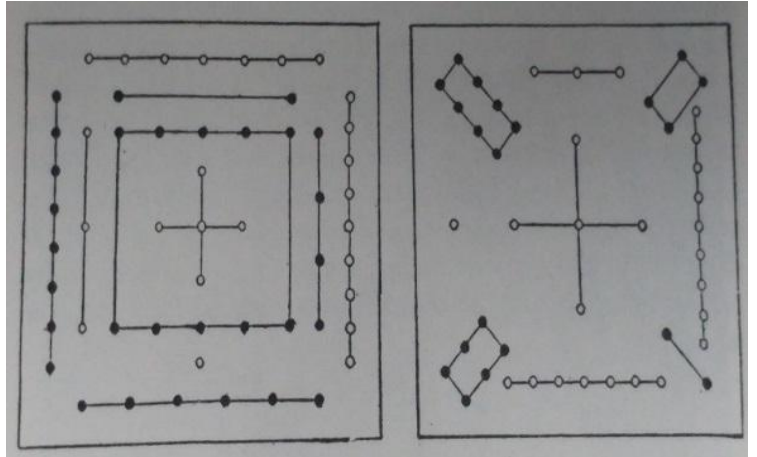

Mainland's knots in strings (Cajori, 1993) [7]

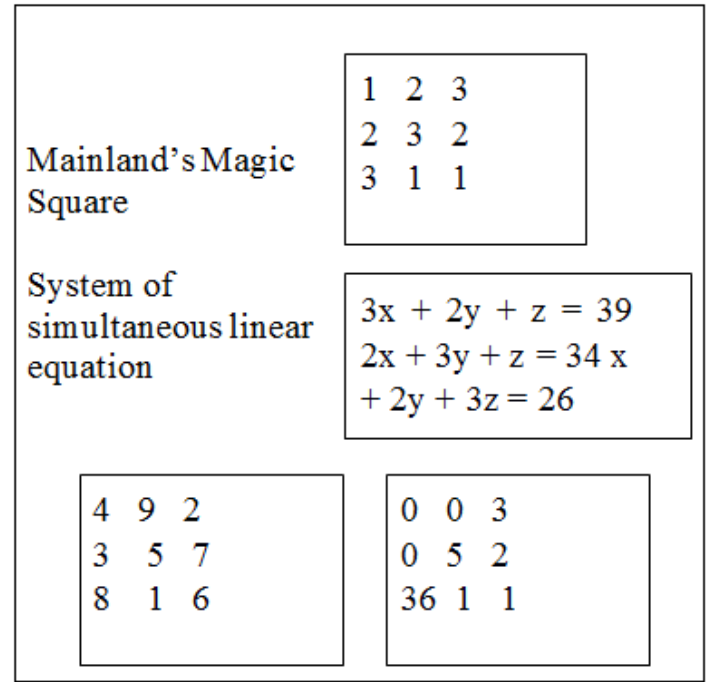

Column operations on the matrix (Merzbach \& Boyer, 2011) [3]
The second of the most well-known numeral system in ancient China is the rod system. In this system, each number notation was represented by a certain number of rods. This eventually transformed into a calculation board, known as the Chinese abacus (suan-pan).

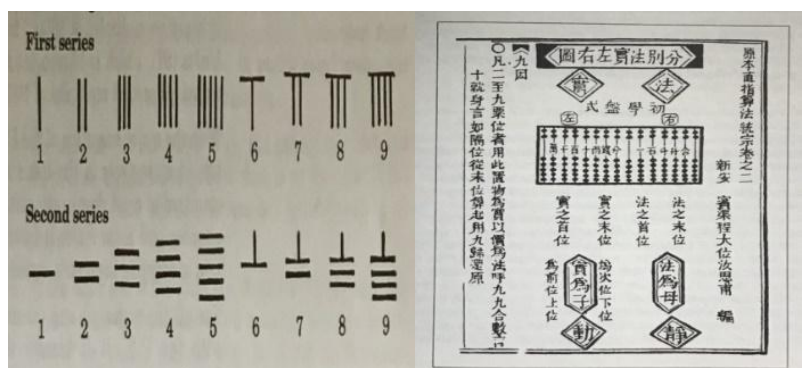

The Mainlanders' Rod Numerals (Gullberg, 1997) [4], Early printed picture of an abacus on the right (Merzbach \& Boyer, 2011, p.179) [3]

The last contribution concerned in mathematical symbols from China was the tortoise plastron [6]. This shows a record of mathematics in China as early as the Shang Dynasty (16th - 17th century, B.C.), in the form of inscriptions on tortoise shells and bones. This evidence tells us about China's circular granaries, bronze weapons, and ceremonial vessels, which implies the existence of an exchange economy and currency. These constitutes to the Zhang Dynasty culture (human endeavour or achievements) at that special moment in the history of mathematics. These improvements in agriculture fostered the division of labour, which is a form of Zhang civilisation (rather in spiritual sense but not daily practical like culture).
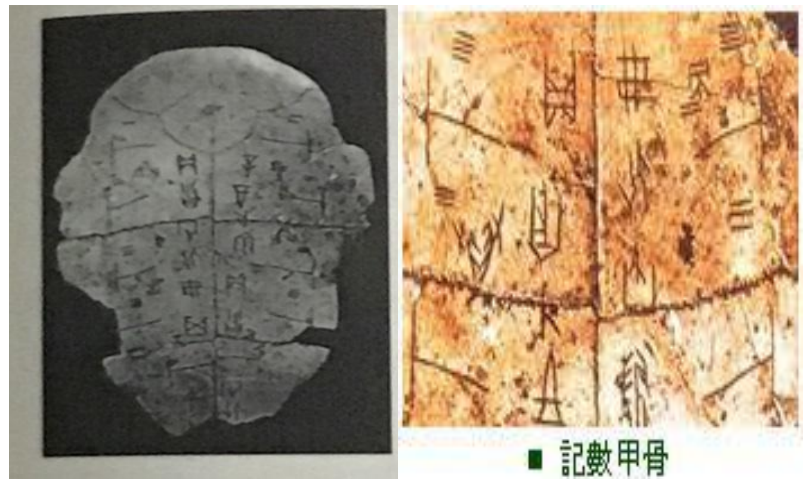

Rather than recording numerals, Zhang Dynasty relics also demonstrate China's culture and civilisation during this era. Pictures come from Katz [6]

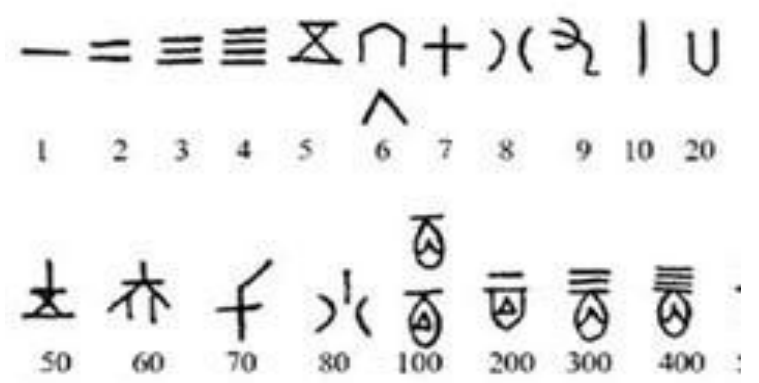




\section{Basic Set and Probability Theories}

When discussing basic set and probability theories, numerous mathematical nota- tions might be used or related. These notations are usually referred to as symbolism. Un- der the use of mathematical logic, a set of sentences can be expressed by using formal language. When it is combined with a deductive apparatus, a formal system can be cre- ated. This is why mathematics is often viewed as a language. The following sections will describe set notations or symbolism in detail, which includes events and sample space, outcome spaces, Venn diagrams, independent events, exclusive events, conditional probabilities, and Bayes' Theorem.
Events, Sample, and Outcome Spaces of a Probability

In probability theory, all the possible outcomes may be grouped into a set, known as a sample space, and is denoted by $\mathrm{S}$ during an experiment is performed. If $E$ is selected as a subset of the sample space $S$, it is called an event space. Set theory is used in probability because it fits basic requirements and modelling, since a set is just a collection of similar objects (a case study is the set of all possible outcomes). The outcome space refers to the set of all results from an experiment. It should also be noted that a trial (or experiment) is an infinitely repeated procedure together with a welldefined sample space.

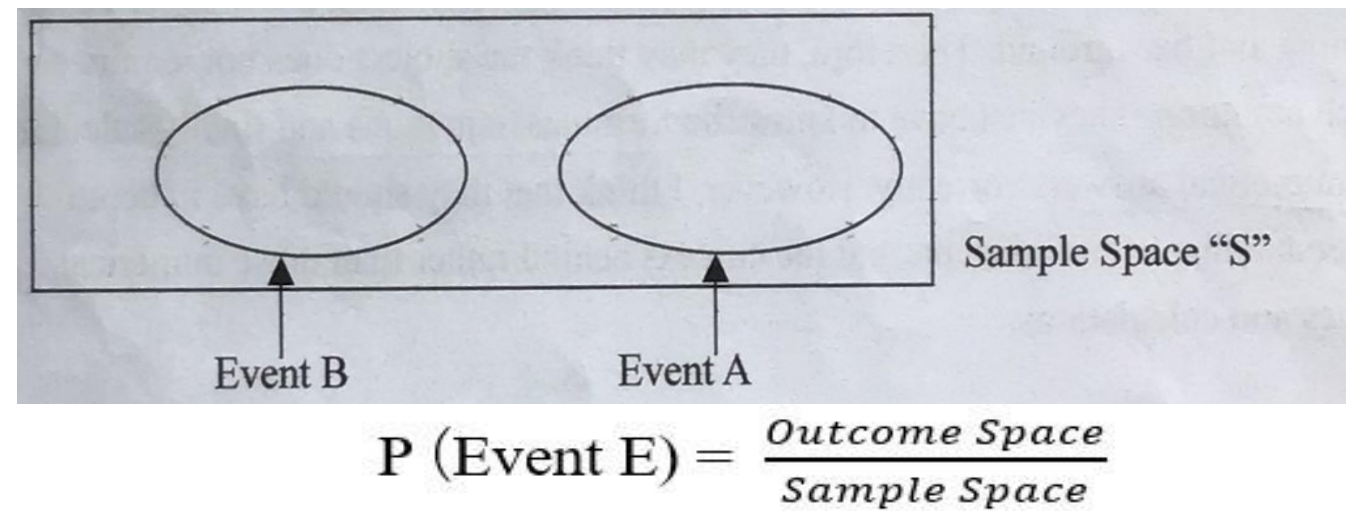

\section{Venn diagram, Independent and Exclusive Events}

As most probability theories are represented by sets, it is natural for a Venn diagram to be used for visualisation. A Venn diagram shows relationships for sets and groups of objects - especially in depicting intersections, minus, exclude and include of sets, etc. For any two events, $\mathrm{A}$ and $\mathrm{B}$ are independent if the existence of A does not affect the existence of B. Or in symbolic form:

$\mathrm{P}(\mathrm{A}$ and $\mathrm{B})=\mathrm{P}(\mathrm{A}) \mathrm{P}(\mathrm{B})$

One case study is the tossing of a coin and getting "heads" together with rolling a dice and getting a five. They are independent of each other.

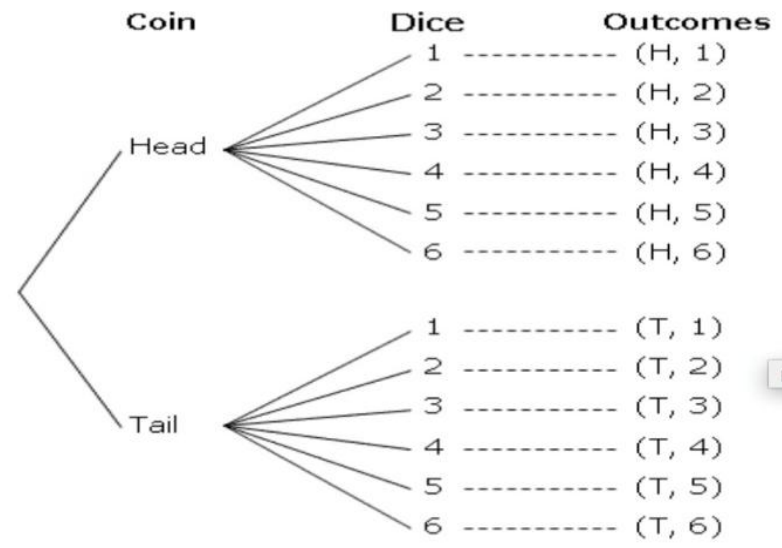

Next, for any two events, $\mathrm{A}$ and $\mathrm{B}$ are mutually exclusively if the occurrence of $\mathrm{A}$ and the occurrence of B cannot happen together. For example, one can either choose to turn left or right, but not both. Or in symbolic form:

$\mathrm{P}(\mathrm{A}$ and $\mathrm{B})=0$

\section{Conditional Probability and Bayes' Theorem}

This usually refers to the conditional probability, as the probability of event $\mathrm{A}$ given that event B has just occurred. It is written in the symbolic form like the following diagram.

$$
\mathrm{P}(\mathrm{A} \mid \mathrm{B})=\frac{P(A \cap B)}{P(B)}
$$

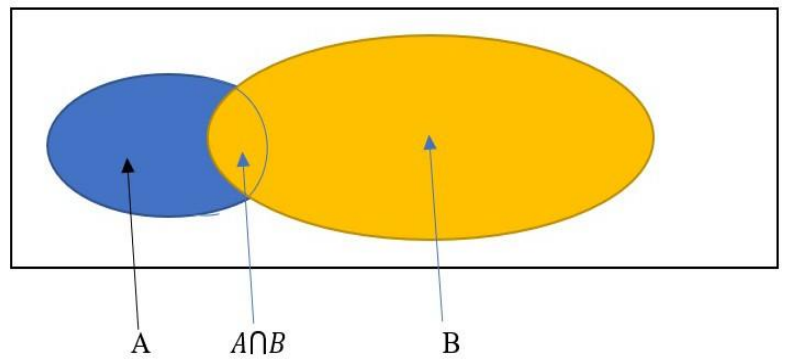

Practically speaking, the probability of rolling a " 3 " in a one-die toss is $1 / 6$. How- ever, when it is given that $B=\{3,4,5,6\}$, then the probability of getting a $\{3\}$ is only $1 / 4$. This is the meaning of conditional probability. Furthermore, if event $\mathrm{A}$ is independent of event $\mathrm{B}$, i.e. $\mathrm{P}(\mathrm{A}$ and $\mathrm{B})=0$, one will have:

$\mathrm{P}(\mathrm{A} \mid \mathrm{B})=\mathrm{P}(\mathrm{A})$ 
Bayes' Formula is often referred to when discussing conditional probability, which states the following:

Let the event $\mathrm{A}$ happened under a hypothesis Hi with a known probability $\mathrm{P}\left(\mathrm{A} \mid \mathrm{H}_{\mathrm{i}}\right)$. Furthermore, assume that the probabilities of hypotheses $\mathrm{H}_{1}, \mathrm{H}_{2}, \ldots$, $\mathrm{H}_{\mathrm{n}}$ are given (known). One may get the conditional probability of $\mathrm{H}_{\mathrm{i}}$ where $\mathrm{i}=1,2 \ldots \mathrm{n}$ such that event $\mathrm{A}$ happened is:

$\mathrm{P}\left(\mathrm{H}_{\mathrm{i}} \mid \mathrm{A}\right)=\left[\mathrm{P}\left(\mathrm{A} \mid \mathrm{H}_{\mathrm{i}}\right) \mathrm{P}\left(\mathrm{H}_{\mathrm{i}}\right) / \mathrm{P}(\mathrm{A})\right.$

Where, $\mathrm{P}(\mathrm{A})=\mathrm{P}\left(\mathrm{A} \mid \mathrm{H}_{1}\right)+\mathrm{P}\left(\mathrm{A} \mid \mathrm{H}_{2}\right)+\ldots+\mathrm{P}\left(\mathrm{A} \mid \mathrm{H}_{\mathrm{n}}\right)$

Or the tree diagram,

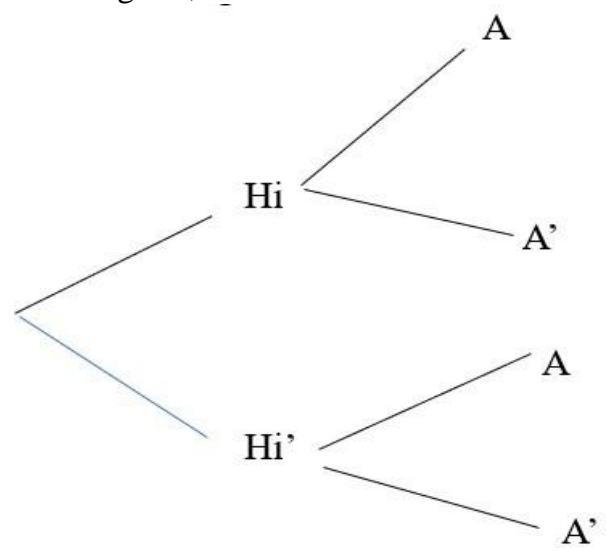

\section{Major Finding for Statistics}

From symbolism, set theory, and basic probability, one can transcend it to explain the butterfly eff ect in terms of mathematical symbols.

\section{Basic Combination and Permutation Theory}

Given a collection of items, if one wants to select them from it where the order is not a matter (not counted), it is called a combination. For instance, this case study is of an apple, an orange and a pear. As such, their combinations are the following: apple, orange; apple, pear; orange, pear. Similarly, considering a subset $\mathrm{E}$, which contains $\mathrm{k}$ distinct elements of a set $\mathrm{S}$,
$\mathrm{E}$ is then called the k-combination of $\mathrm{S}$. While on the other hand, the number of k-combinations of a set $n$ is equal to:

$$
\left(\begin{array}{l}
\mathrm{n} \\
\mathrm{r}
\end{array}\right)=\frac{\mathrm{n}(\mathrm{n}-1) \ldots(\mathrm{n}-\mathrm{r}+1)}{\mathrm{r}(\mathrm{r}-1) \ldots 1}
$$

If a sequence (or order) is rearranged, where the order is taken into account, it is named as a permutation (without replacement) $-\mathrm{n}^{\wedge} \mathrm{r}$. Furthermore, after each selection of fruit is replaced (taken away), the result is: apple, orange; apple, pear; orange, pear; orange, apple; pear, apple; apple, pear or 6 .

${ }^{n} P_{r}=\frac{n !}{(n-r) !}=n(n-1) \ldots(n-r+1)$

\section{What is the Butterfly Effect?}

The term was first introduced by meteorologist Edward Lorenz. The butterfly eff ect explains that a small change can lead to much greater (serious) consequences over time.

\section{Catching the Butterfly: A Foreseeable Prediction}

Consider the following imaginary statistical experiment that is often used when teaching statistics:

One should first toss three (or more) coins together in front of the participants, such that heads are on the front and tails are on back. The participants are then required to list all possible outcomes after tossing the coins.

In general, the set of all feasible results (outcomes) would be:

$\mathrm{S}=\{\mathrm{HHH}, \mathrm{HHT}, \mathrm{THH}, \mathrm{HTT}, \mathrm{THT}, \mathrm{TTH}, \mathrm{TTT}\}$ (suppose one will obtain such expected result). There are also other combinations and permutations of heads and tails when each of them are assigned with a number. Hence, the outcome space is not just unique.)

Finally, the participants must count the number of tails for each possible outcome and then list them on a table, as seen below:

\begin{tabular}{|l|c|c|c|c|c|c|c|c|}
\hline Possible Outcomes $\left(\mathrm{s}_{\mathrm{i}}\right)$ & HHH & HHT & HTH & THH & HTT & HTT & TTH & TTT \\
\hline No. of tails obtained $\mathrm{t}_{\mathrm{i}}$ & 0 & 1 & 1 & 1 & 2 & 2 & 2 & 3 \\
\hline
\end{tabular}

(Clearly, the above table does NOT list all possible outcomes for tails.)

A function $\mathrm{f}$ (which should be called the random variable) must also be considered. Intuitively, $\mathrm{f}$ maps all the possible outcomes $\left(\mathrm{s}_{\mathrm{i}}\right)$ to the number of tails $\mathrm{T}_{\mathrm{i}}$

$\mathrm{f}_{\mathrm{i}}: \mathrm{s}_{\mathrm{i}} \longrightarrow \mathrm{t}_{\mathrm{i}}$ (i.e. no. of tails obtained as it takes values $\mathrm{t}$ $=0,1,2,3 \ldots$ by HKU, Dr. K.T. Leung's lecture note 1993-1994)
Or strictly speaking $\mathrm{f}: \mathrm{S} \longrightarrow \mathrm{T}$ (i.e. $\mathbf{R}-$ Real Number)

This is just a concept/definition of random variables. One must first group the outcome space into different categories and then map it onto the "feasible values of the random variables". In normal circumstances, these values are real numbers, but there may also be other types of elements. These "special values of a random variable" are thus called "random elements". 
It should be noted that the random variable described is not the only choice, it depends on the mapping domain and its couple. Therefore, there may be a countable finite number of random variables, as mentioned above. The collection of all random variables is called "random function" $-F(X)$, and in addition, implies the "stochastic process" for handling randomness.

If one maps each value of categorised random variables into their own probability, the corresponding probability function can be formulated. When the occurring events are mutually exclusive, Bayes' Theorem can be applied to reveal the conditional possibilities of the corresponding elements in a series of sample space. Conversely, if these events are not mutually exclusive, the probability reduces to only a direct multiplication of two probabilities. The method to test the independence can only be through a numerical result, whether $\mathrm{P}(\mathrm{B} \mid \mathrm{A})=\mathrm{P}(\mathrm{B})$. Hence, one can make those necessary decisions (or even predictions).

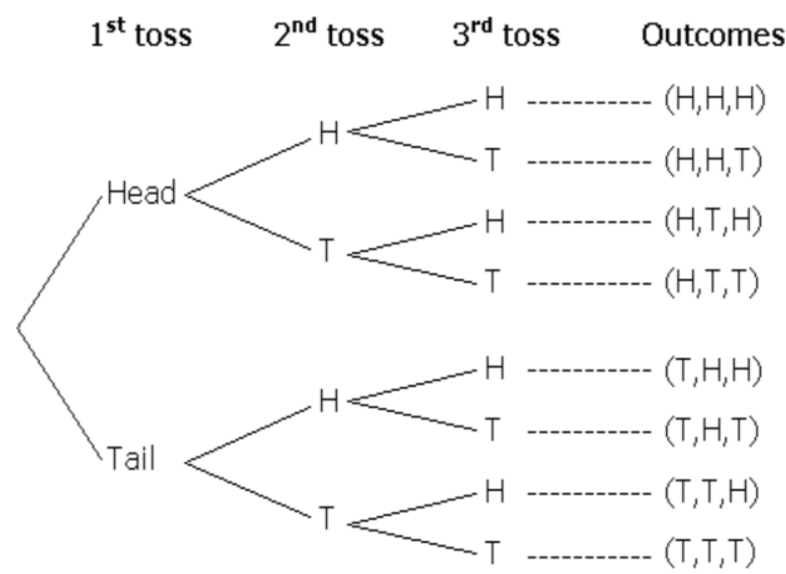

In the above tree diagram, we group the result of tossing one tail and then assign it to random variable one; tossing two tails is assigned to random variable two and three tails to random variable three. The advantage of such mapping is that there are four catalogues with the probabilities: $1 / 8,3 / 8,3 / 8$, and 1/8 respectively. By applying conditional probability and Bayes' Theorem, one may guess in a reversing order about the initial chance of an event, together with a series of feasible chained outcomes. Furthermore, if all the mapped random variables can be found, every final consequence will be known.
This research attempts to investigate the following: classifying all events that occur with similar properties (in the outcome space — such as same outcomes without considering order) and forming groups or catalogues. Then they are mapping onto an individual random variable correspondingly. Every corresponding domino effect (or individual event) will be included (or caught). In addition, with the use of Bayes' Theorem, one may even look backwards to find the conditional probability of an event. Indeed, the random variable acts as a bridge that connects domino effects and the former experiment (chaos') sample space. With the probability tree diagram (which will be described in the following section), all feasible probabilities can now be applied to those random variables to initiate a series of chain reactions, which lead to final outcomes. Hence, the "randomness" can now be calculated or even forecasted.

\section{The Domino Effect}

After mapping the random variable, one may intuitively map each $t_{i}$ into a category $\mathrm{ca}_{\mathrm{i}}$ with same range $r$ of marks such as " 0 - 50"; "51 - 60"; "61 - 70"; "71 - 80"; "81 - 90"; "91 - 100" that stepping wisely, then:

$\mathrm{e}_{\mathrm{i}}: \mathrm{t}_{\mathrm{i}} \longrightarrow \mathrm{ca}_{\mathrm{i}}$

or strictly speaking:

$\mathrm{E}: \mathrm{T} \longrightarrow \mathrm{CA}$

(Case Study: Any school student's examination marks must follow into either one of these categories.)

If one continues to map each of these $\mathrm{ca}_{\mathrm{i}}$ with traditional "select...case..." programming statement to give out those decisions $\mathrm{d}_{\mathrm{i}}$ like grade "F"; " $\mathrm{E}$ "; " $\mathrm{D}$ "; "C"; "B"; "A" followed by predictable events pe such as "fail"; "marginal pass"; "fair pass"; "good"; "very good"; "Excellent" computing algorithm, one will get:

$$
\begin{aligned}
& \mathrm{sc}_{\mathrm{i}}: \mathrm{ca}_{\mathrm{i}} \longrightarrow \mathrm{d}_{\mathrm{i}} ; \\
& \mathrm{de}_{\mathrm{i}}: \mathrm{d}_{\mathrm{i}} \longrightarrow \mathrm{pe}_{\mathrm{i}} \longrightarrow
\end{aligned}
$$

or strictly speaking:

$$
\mathrm{SC}: \mathrm{CA} \longrightarrow \mathrm{DE} \text {; }
$$$$
\mathrm{DE}: \mathrm{D} \longrightarrow \mathrm{PE}
$$

The above calculations are established to describe the following relationships:

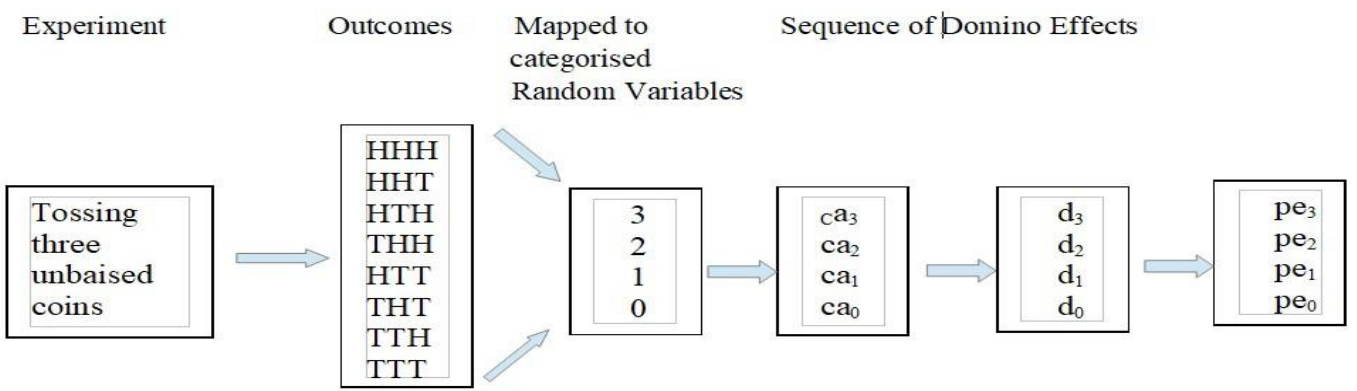

Fig-2: The mapping of random variables which may predict feasible outcomes 
The above calculations are established to describe the following relationships:

a) Probability outcome spaces and real numbers;

b) Real numbers and different categories;

c) Categories and consequences.

Different categories and decisions (in this case, student grades) followed by predicable events whether a student passes or fails and what proposed actions should be taken.

This case study maps all non-linear and nondeterministic feasible outcomes (possibilities) onto something like real numbers (i.e., finding all possible random variables). A series of domino effects or one-toone mapping (see Fig-2) will be formed. As a result, linear phenomena and conjugate cases can be predicted. More specifically, one can list/find all consequences and domino effects that might eventually occur due to the butterfly effect. I name such kind of methodology which catch the "Butterfly Effects" as the HKLam ('s Net-Seizing) Theory.

That said, there may be some errors when attempting to predict the results. This is due to the countless random variables that exist in a special nondeterministic event. Hence, one may use both fuzzy' logic and expected values to solve those unexpected events that occurred problems.

In brief, probability and combinatorics will be used to list all possible outcomes. The corresponding consequences will also be mapped with random variables, together with the previously probability. The figure in the next page follows is suggesting a generalised case for such an effect. Alternatively, by associated with a list of domino effects that mapped alongside suitable grouped random variables, the conditional probability of a related event to occur can be revealed. One case study is the forecasting typhoons in Hong Kong during autumn, where the weather system is complex. High-pressure systems, northeast monsoon winds, west wind-range effect, together with Fujiwhara's Effect, influences these predictions. When these factors are considered as domino effects and mapped alongside different grouped random variables, the corresponding conditional probability might be achieved. However, these results may not be accurate as weather patterns fall into the category of a chaos sytem. As such, forecasting probability must be the primary focus, as opposed to the real outcome.

https://hk.news.appledaily.com/local/realtime/article/20 $180908 / 58642539$

https://hk.news.appledaily.com/local/daily/article/20180 $729 / 20463444$

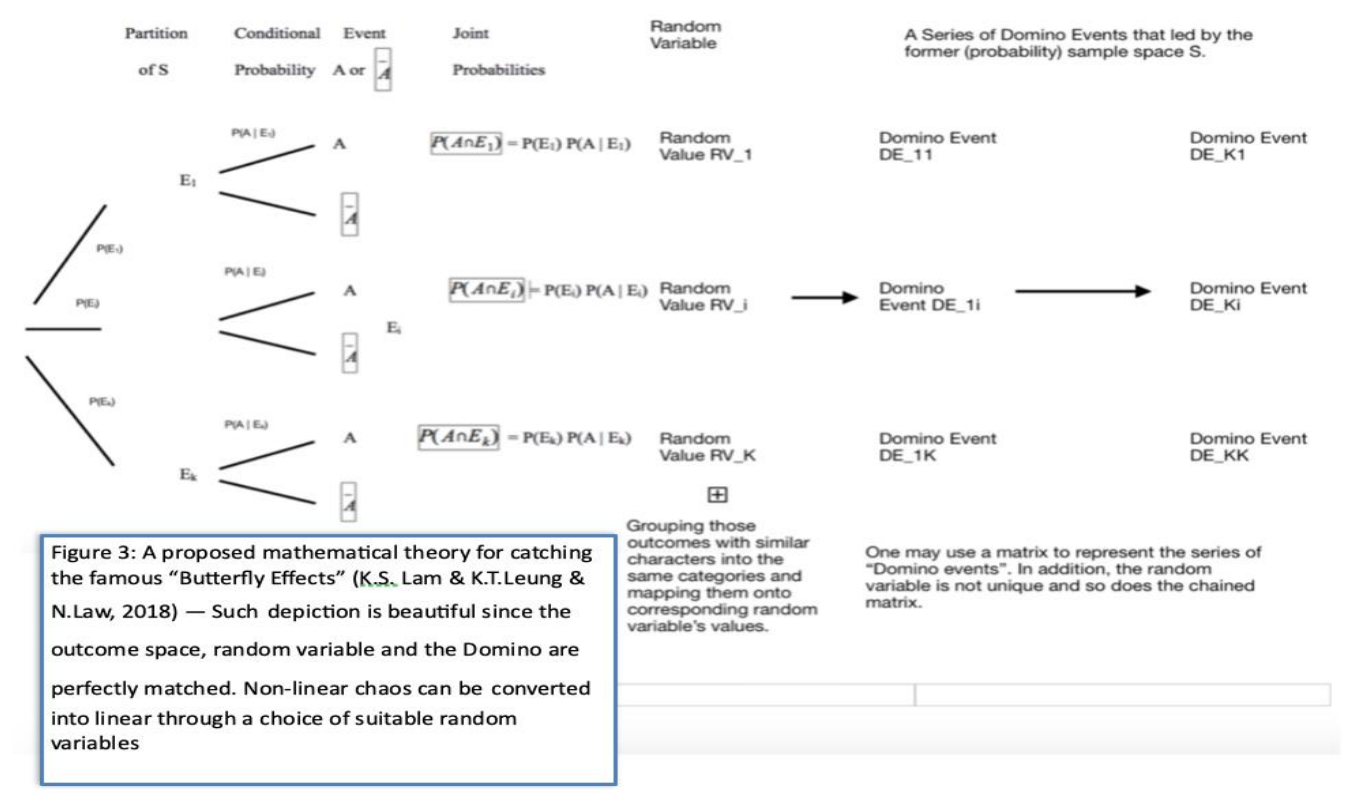

\section{Significance of Research}

One of the significant contribution of this research is that it initialises the research of my proposed butterfly effect theory. This is because different random variables are able to map all feasible outcomes with a predictable output result. Therefore, all outcomes that correspond to the butterfly effect can be caught (included) in any one of the predicted outcome domino cases. These findings are only the beginning of such research. Furthermore, there are many applications from this study, which are listed in the following: 


\section{Cutting "Tofu" for better data storage;}

How should tofu be with fewer cuts in order to achieve the best number of sub-cubes outcomes? Such kind of cutting Tofu is indeed a random process (which is a non-linear and non- deterministic one) where people may find the best efficient cutting for the same amount of data storage. Each feasible cutting method corresponds to an individual probability. Different types of data storage may corresponds to different groups of cutting methods. These routes are respectively corresponding to various grouped random variables. The best possible result with the most efficient capacity and shortest cutting routes can then be calculated. Moreover, to practically solve this problem, the combinatoric and inclusion-exclusion principle together with systems of distinct representatives will be needed to find all of the cutting routes. Hence, through my butterfly philosophy, one can easily find the best minimum cuts in order to maximise the data storage. Even in the case of data searching, it can be made faster since the best route findings are easier. This can help us establish a future digital library. From mathematical representation theory, the corresponding one is social representation theory, which implies the existence of different school cultures and the diversity.

\section{Building a quantum computer chip with musical resonance;}

One can even build a quantum computer chip with suitable resonance as the control. This might be possible as both the quantum spin and their entanglement are unpredictable in normal situations. That is why these spins and entanglements are nondeterministic and non-linear. As a result, this study's statistical method might be able to predict all feasible outcomes of the spin and entanglement. Furthermore, if musical resonance can be created to control the spins and entanglements, one can even build quantum computer chips in a practical manner.

\section{Predicting customer behaviour;}

Through this study's statistical random variables, one might be able to predict customer behaviour, as these behaviours are also nondeterministic and non-linear. Hence, all outcomes and consequences are able to be predicted.

\section{Making the best decisions for events to occur in the multiverse;}

The multiverse has many possible choices. By employing this study's butterfly effect philosophy, all possible consequences of an event can be revealed. The best route can then be chosen according to the different probabilities and chained consequences.

Establishing a management system to control different home-used radio waves; Today's homes have many electro-magnetic waves such as infra-red, wi-fi,
$\mathrm{RF}$, and Bluetooth etc. By introducing a management information system (MIS) (together with a password) to assign each electro-magnetic wave with a suitable channel, one can login into an individual network system, one at a time, without causing any interference. Conversely, with similar MIS, electronic devices can be introduced (such as D1 min-pro) to collect electromagnetic waves. Although the received electronic signals are in square form, by adding suitable electronic circuits with suitable magnifying effects and smoothing properties, it feasible to use capacitors and chargeable batteries to store electrical energy back in achieving our daily usage consumption.

\section{CONCLUSION}

In conclusion, symbolism is closely connected with modern mathematics. As previously mentioned, mathematics symbols first appeared as visual objects, such as animals. These primitive symbols were conceptualised to form numeral symbols, which were then simplified into notations. The collection of these symbols may form words. Mathematics can thus be seen as a type of language as it has language-type properties such a grammar. One may refer this as the formal language or linguistic. Furthermore, it provides quantitative rational foundations (a kind of instrument) for different subjects like physics etc. This constitutes to a linking, which relates to mathematics. As such, someone considers mathematics as the language of science. One may even consider mathematics as a 'public servant' to these subjects. Obviously, when one views from history, one must relate its connections with human endeavours. These achievements are naturally be included as part of the subject where mathematics is a fraction of our culture. In other words, our culture is composed of human endeavour which is well-connected with mathematics. Therefore mathematics is a part of culture. The similar situation does apply in the civilisation as it is only defined by the spiritual section of the relationships. As mentioned before, there are human activities/achievements, which in turn were viewed as symbolic representations or cultural symbols. Indeed, these human endeavours, which reflect the spiritual, social, customs, and values etc. at that particular historical moment show the civilisation of the country. As mathematics is developed naturally all around our world and reflected back in different civilisations, it is naturally to think that the subject is the common area (or language) of our human to depict our knowledge in every era. It is obviously that the aim of mathematics is developed to help us discover our universe or even implies the existence of only God, Jehovah who sends us the gift. In other words, without mathematics, our world will not be such rationalised and the order will not be discovered. Human endeavour will not be important and there would be no more modern civilisation as nowadays. 
Quantitative (rational) instrument of different subjects through symbols links various topics - A World's common (language) that depicts our world

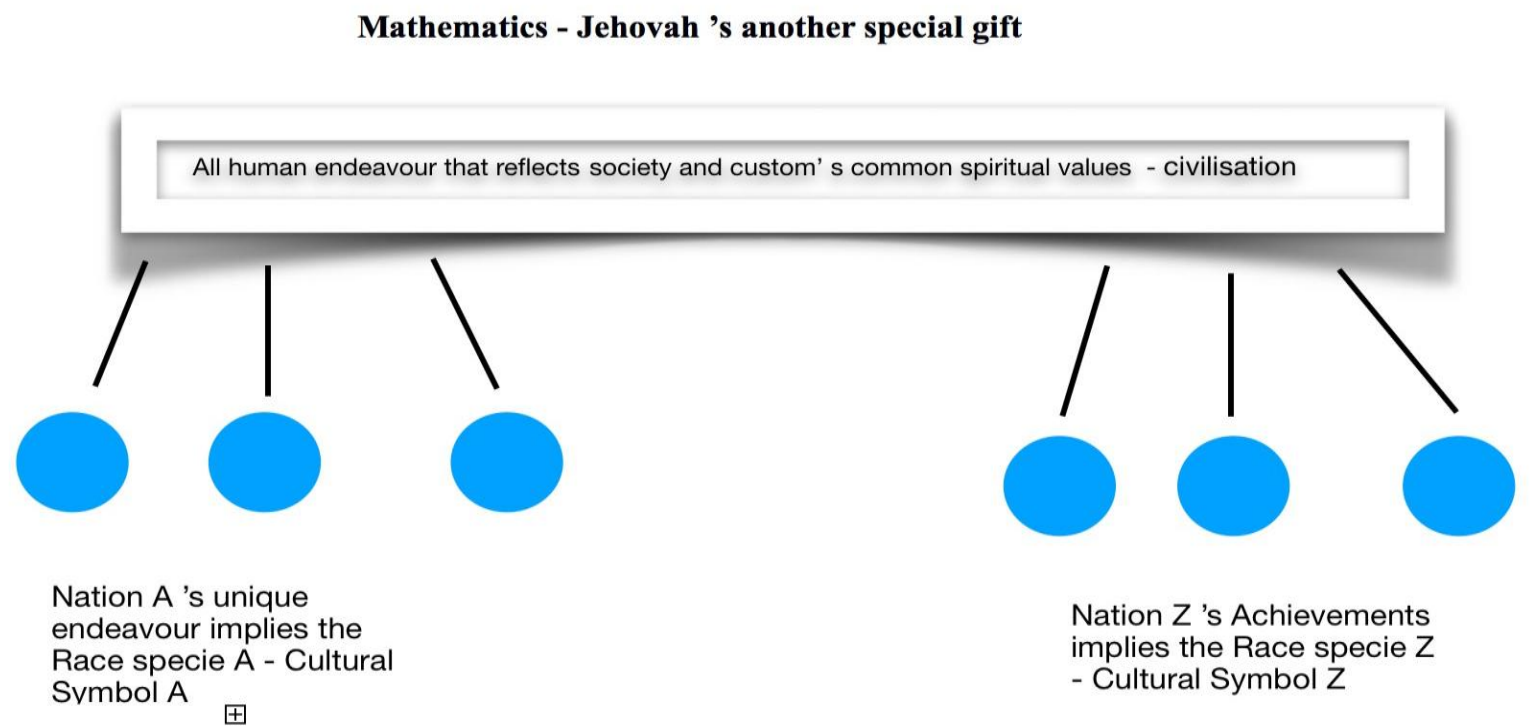

Unique Human ancestor

Self-Owned God: Yahveh

The relationship diagram that links with mathematics and cultures and civilisation. It also implies the existence of unique God, Yahveh

\section{Remarks}

1. From the above discussion, mathematics is highly related to symbolism. To go ahead a step, Kharde in 2016 even suggested that mathematical thoughts, methods and facts can be expressed in terms of symbolism but not in English [8]. Indeed, Mathematics may be a type of language art. This is because art should consist of language and literature. According to Kharde, mathematics is a language that can be read and written in the form of symbols. At the same time, it is easy to find there are lots of mathematical literature which are well descriptor of various mathematics concepts. My conclusion is mathematics can be a subject of language art. With reference to the aforementioned in the previous sections of this paper, mathematics is connected with different subjects. Therefore, my suggestion is to view mathematics as the Federal States with those core axioms, theories and laws as the central government and linked to the other states various subjects with some common areas respectively.

2. From the present paper, we discover that the evolution of language is transformed from elementary signals to symbols. To go ahead a step, symbols can be mapped to the perceptual systems. According to Zhirenov in 2016, there are several methods to determine the category of symbols in the linguistic-cognitive research. The study shows that symbolisation in a language will affect the role of the national-cultural-cognitive association. The result may be difficulties in the formation of a unified concept and image of the world language. Then we may once again introduce the Symbol Interdependency Hypothesis that argues:

A. language encodes perceptual information;

B. Users rely on language statistics in the cognitive process, Hence, we can map language to a perceptual system [9]. However, perception is closely connected to the sensory system that responds to the environment. There is a Butterfly Effects existing in the brain during the process. Therefore, it is interesting to apply my HKLam Theory to catch such kind of Butterfly. Or in the field of translation, we may also net-seize the butterfly of language context uncertainty [10].

3. When one is considering the natural number series 11]: $0,1,2,3, \ldots, \mathrm{n}, \mathrm{n}+1, \ldots$, questions may be raised - What do we mean by "number", or " 0 " or "1" [11]. In addition, when one begins from 0 , then all of the natural numbers can be reached by repeatedly "adding 1" to achieve. Similarly, how do we define the term "adding" and "repeated". By continuously defining these terms through the definition of other more basic terms, one can get the meaning of the abstraction for the natural numbers. But there are still some terms that can be viewed as intelligible without definition or the true 
starting point for the wanted terms' definition. Historically, it was Pythagoras who proposed everything can be deduced from numbers or the so-called "arithmetician" of mathematics. Indeed, the Pythagoras theorem depicts the relationship between the triangle's sides was finally solved with the help of the reduction from arithmetic to logic. Actually, my discovery of the Babylonian about the Pythagoras theorem is from the perspective of Linguistics and Anthropology but NOT the philosophical one. In the case of natural numbers, traditional pure mathematics was first reduced to theory and then performed the logical analysis to get the smallest set of premises and undefined terms from its derivation. This event was done by Peano that the theory of natural numbers could be derived from three primitive ideas and five primitive propositions [12] together with logic [11]. The aforementioned about the mathematical philosophy of natural numbers denote the remarkable beginning in our history but not the technical one.

4. To go a further step, one may connect this paper with the linguistic component of the computational theory of mind (CTM) [13] or the related mental process. Indeed, this paper is highly linked to the symbolic computation or the algebraic computation. This means one may develop the corresponding software or algorithm for the manipulation of the mathematical expressions or the objects. Rather than the algebraic one, the HKLam theory in this paper can be further advanced into the topic probabilistic computation. The present thesis is just the combination of algebraic and probabilistic computation.

5. From the non-theoretically perspective, symbolism is indeed the earliest form of natural language processing (NLP). We also have statistical and neural types. NLP is actually concerned with the interaction between computers and our human (natural) evolved languages such as English, Mandarin, French, Cantonese and Germany etc. To be more specific, NLP means the way in how our computer scientists program the computers to process and analyse large amount of natural language data. One of the case studies of application is our automated telephone (or online) assistant that can provide customer service for an enterprise. Indeed, when symbolism is mixed with linguistics, we have symbolic linguistics representation. If one go a further step, we get the computational linguistics which is just consisting the Natural Language Processing.

\section{REFERENCES}

1. Mazur, J. (2014). Enlightening Symbols: A Short History of Mathematical Notation and Its Hidden Power, Princeton University Press; Princeton and Oxford.

2. Piercy, J. (2013). Symbols: A Universal Language; Michael O’ Mara Books Ltd.

3. MerzBach, U. C., \& Boyer, C. B. (2011). A History of Mathematics, Third Edition. Wiley Press.

4. Gullberg, J. (1997). Mathematics: From the Birth of Numbers, Norton \& Company.

5. Swetz, F. (1994). From Five Fingers to Infinity: A Journey through the History of Mathematics.

6. Katz, V. (2007). The Mathematics of Egypt, Mesopotamia, Chi-na, India, and Islam, Princeton University Press, Princeton and Oxford.

7. Cajori, F. (1993). A History of Mathematical Notations: Two Volumes Bound As One; Dover Publications, Inc, New York.

8. Kharde, U. (2016). The Symbolic Language of Mathematics, The Explorer: A multidisciplinary Journal of Research; ISSN : 2456-0995

9. Louwerse, M. M. (2018). Knowing the Meaning of a Word by the Linguistic and Perceptual Company It Keeps, Topics in Cognitive Science, DOI: $10.1111 /$ tops. 12349

10. Zou, X. (2017). Understanding: How to resolve Ambiguity, https://www.researchgate.net/publication/3200493 56

11. Ronald, C. (1982). Classic of Mathematics, Prentice Hall Publisher.

12. The three primitive ideas in Peano's arithmetic are: 0 , number, successor. The five primitive propositions are: 1) 0 is a number. 2) The successor of any number is a number. 3) No two numbers have the same successor. 4) 0 is not the successor of any number. 5) Any property which belongs to 0 , also holds for the successor as well as all numbers.

13. https://plato.standford.edu/entries/computationalmind/ 